\title{
28 Research Square \\ Efficacy and Safety of Keluoxin Capsule in the Treatment of Diabetic Kidney Disease
}

Jingxin Zhou ( $\nabla$ zhoujingxin2000@126.com )

Beijing University of Chinese Medicine https://orcid.org/0000-0003-3734-9778

\section{Wenhua Zhang}

Beijing University of Chinese Medicine Affiliated Dongzhimen Hospital

\section{Jie Yang}

Beijing University of Chinese Medicine Affiliated Dongzhimen Hospital

\section{Qiaoqiao Liu}

Beijing University of Chinese Medicine Affiliated Dongzhimen Hospital

\section{Yizhen Lu}

Beijing University of Chinese Medicine Affiliated Dongzhimen Hospital

Jian Jin

Beijing University of Chinese Medicine Affiliated Dongzhimen Hospital

\section{Guizhen Miao}

Beijing University of Chinese Medicine Affiliated Dongzhimen Hospital

\section{Yanbing Gong}

Beijing University of Chinese Medicine Affiliated Dongzhimen Hospital

\section{Protocol}

Keywords: Keluoxin capsule, d iabetic kidney disease, protocol, systematic review, meta-analysis

Posted Date: December 20th, 2021

DOI: https://doi.org/10.21203/rs.3.rs-1111126/v1

License: (c) (1) This work is licensed under a Creative Commons Attribution 4.0 International License.

Read Full License 


\section{Abstract}

Background: Diabetic kidney disease (DKD), which is a serious complication of diabetes and the leading cause of end-stage renal disease, becomes a major health concern worldwide. Keluoxin capsule, a Chinese patent medicine used for DKD, has been widely used in diabetic kidney disease, but its efficacy and safety have not yet been clarified. Therefore, the aim of this systematic review and meta-analysis is to summarize the efficacy and safety of Keluoxin capsule in the treatment of DKD.

Methods: A systematic literature search will be conducted in PubMed, Cochrane Library, EMBASE, China National Knowledge Infrastructure, WANFANG database, VIP, SinoMED, and Chinese Biomedical Literature Database (CBM) to ensure all possible randomized controlled trials (RCT) studies on K eluoxin capsule to November 1, 2021. The primary outcome to be assessed will include the change in albuminuria and estimated glomerular filtration rate (eGFR), while secondary outcomes will be serum creatinine, blood urea nitrogen, glycated hemoglobin, total cholesterol, low-density lipoprotein cholesterol, and triglycerides, etc and adverse events. The quality of the included studies and the risk of bias will be independently assessed by two reviewers using the risk of bias assessment tool from Cochrane Handbook. We will conduct random-effects model meta-analysis using Review Manager software (Revman5.3).

Discussion: This systematic review and meta-analysis will objectively evaluate the effect of Keluoxin capsule for DKD, and provide evidence for Keluoxin capsule in the treatment of DKD.

Ethics and dissemination : Ethics approval is not required for this study. We aim to publish the results of this systematic review in a peer-reviewed journal. INPLASY registration number: INPLASY 2021110067.

\section{Introduction}

Epidemiological studies show that diabetes mellitus (DM) currently affects more than 463 million people globally, and the number of individuals with DM is estimated to rise up to 578 million by 2030 , and 700 million by 2045 [1]. The consequential prevalence of microvascular and macrovascular diabetes complications also rapidly increases. Diabetic kidney disease (DKD), which is a serious complication of DM defined as persistent albuminuria or reduced estimated glomerular filtration rate (eGFR), affects 30$40 \%$ individuals with diabetes and approximately $50 \%$ of them can progress to end-stage renal disease (ESRD) [2]. Thus, the improvement of health outcomes for DKD needs to refocus on the strategies for controlling the progression of DKD to the ESRD.

Improving albuminuria and reduced eGFR in DKD patients has become an important treatment to delay the progress to ESRD [3]. Several cross-sectional studies have shown an increasing use of glucoselowering medications, RAS blockers, and statins, resulting in a progressive improvement in glycemic, blood pressure and lipid control, leads to the reduction in the prevalence of albuminuria and the increment in the prevalence of reduced eGFR [4]. However, these therapies for DKD also lead to an increased risk of significant side effects such as hyperkalemia associated with RAS blockade [5], genital 
mycotic infections, hypoglycemia, and diabetic ketoacidosis caused by SGLT2 inhibitors [6], suggesting that additive effects may also cause harm.

Chinese medicine has been shown a great beneficial effect on reduction of albuminuria and renal function, and safe in the treatment of DKD [7]. Keluoxin capsule, a Chinese patent medicine used for DKD, is composed of Astragalus membranaceus, Ligustrum lucidum, leech, Rheum officinale, Radix Pseudostellariae, and Lycium barbarum. Clinical studies have been demonstrated that Keluoxin capsule can improve proteinuria and protect renal function in the DKD patients without obvious side effect [8]. At present, there are many clinical trails of Keluoxin capsule, but its efficacy and safety for DKD have not been objectively evaluated. Therefore, we will conduct a systematic review and meta-analysis evaluating the benefits and harms of Keluoxin capsule in the patients with DKD to provide trustworthy evidence for clinical use.

\section{Methods}

\subsection{Study registration}

This protocol has been registered on INPLASY (ID: INPLASY 2021110067) and will be conducted based on the Preferred Reporting Items for Systematic Reviews and Meta-Analysis Protocol statement guidelines [9]. Because all the research materials are published studies, this study does not require ethical approval.

\subsection{Inclusion and exclusion criteria for this review 2.2.1 Types of studies}

Only randomized controlled trials (RCTs) will be eligible for inclusion regardless of the languages. It will not include animal experiments, case reports, non-clinical researches, commentaries, repeated publications, etc RCTs with incomplete and unavailable important data will be excluded.

\subsubsection{Type of participants}

The study will include adult participants aged 18 years or older who are definitely diagnosed with DKD according to Kidney Disease Outcomes Quality Initiative (KDOQI) criteria [10]. There will be no restrictions on the type of DM, stage of the DKD, gender, nationality, race, education, and job.

\subsubsection{Type of interventions}

We will take into account all intervention trails that meet inclusion criteria and include treatment with Keluoxin capsule based on the control without limit of the dosage or course. The comparators could consist of any western medicine, placebo, or no intervention. Both groups received the same conventional treatments of DKD, including comprehensive management of glycaemia, blood pressure, serum lipid level, life-style and nutrition in accordance with Kidney Disease Outcomes Quality Initiative clinical practice 
guidelines' recommendation. Any herbals or Chinese medicine treatment will be excluded from the analysis.

\subsubsection{Primary and secondary outcomes}

The primary outcome will be the mean change in albuminuria and eGFR $\left(\mathrm{mL} / \mathrm{min} / 1.73 \mathrm{~m}^{2}\right)$. Albuminuria indicators will include urinary albumin/creatinine ratio (UACR, $\mathrm{mg} / \mathrm{g}$ ), urine albumin excretion rate for 24 $\mathrm{h}$ urine collection $(\mathrm{g} / 24 \mathrm{~h})$, or micro albuminuria $(\mathrm{mg} / \mathrm{L})$.

Secondary outcomes will be kidney function (serum creatinine $[\mu \mathrm{mol} / \mathrm{L}]$ and blood urea nitrogen [mmol/L]), glycated hemoglobin $\left(\mathrm{HbA}_{1 \mathrm{c}}, \mathrm{mmol} / \mathrm{L}\right)$, lipid indicators (total cholesterol [mmol/L], low-density lipoprotein cholesterol [mmol/L], and triglycerides [mmol/L]); additional cardiovascular outcomes are systolic and diastolic blood pressure $(\mathrm{mm} \mathrm{Hg})$, and heart rate $(\mathrm{bpm})$ from baseline.

The safety outcomes will be related to the number of patients with Keluoxin capsule to have adverse events.

\subsection{Search strategy}

PubMed, Cochrane Library, EMBASE, China National Knowledge Infrastructure, WANFANG database, VIP, SinoMED, and Chinese Biomedical Literature Database (CBM) will be searched to ensure all possible RCT studies on Keluoxin capsule, without limitation of the language. The time interval for literature searching will be from inception of the library to November 1,2021. The key search terms will be composed of diabetic kidney disease or diabetic nephropathy, Keluoxin capsule, and Randomized Controlled Trial. A search strategy example in PubMed is shown in Table 1.

Table 1

\begin{tabular}{|ll|}
\hline Number & Search terms \\
\hline 1 & diabetic kidney disease [MeSH] OR diabetic kidney disease OR diabetic nephropathy \\
\hline 2 & \begin{tabular}{l} 
keluoxin capsule OR ke luo xin capsule OR ke-luo-xin capsule \\
randomized controlled trial [MeSH] OR clinical trails OR randomized OR randomly OR \\
\hline 4
\end{tabular} \#1 AND \#2 AND \#3 \\
\hline
\end{tabular}

\subsection{Study selection}

The records will be extracted from each study and imported into EndNote X9, while duplicates will be removed. Two independent reviewers will screen titles and abstracts to exclude irrelevant studies and examine full texts according to the inclusion criteria/exclusion criteria. Disagreements between the two reviewers will be resolved by a third reviewer. Additionally, references of included studies will be searched for further potentially relevant articles. If a study was published in duplicate, we will include the report containing the most recent and comprehensive information such as the largest sample size. 


\subsection{Data extraction and analysis}

\subsubsection{Data extraction}

Two reviewers will independently extract the following information from the selected studies with a predefined form: first author, year of publication, country, study design, sample size, gender, age, stage of DKD, dose and course of Keluoxin capsule, type of control, type of outcome and assessment. If the information is missing or unavailable to be extracted directly from the articles, we will contact the corresponding authors to obtain the data. The studies will be excluded if we were unable to obtain relevant data. All contacts with authors will be documented.

\subsubsection{Risk of bias assessment}

The methodological quality of the included studies and the risk of bias will be independently assessed by two reviewers using the risk of bias assessment tool from Cochrane Handbook [11]. The sources of bias assessment will include selection bias (random sequence generation and allocation concealment), performance bias (blinding of participants and personnel), attrition bias (incomplete outcome data), reporting bias (selective reporting), and other bias sources. Any disagreement will be resolved by the third reviewer.

\subsubsection{Data synthesis and analysis}

We will conduct random-effects model meta-analysis using Revman software (version 5.3, Copenhagen: The Nordic Cochrane Center, The Cochrane Collaboration). The primary and secondary outcomes will be estimated as continuous outcomes evaluated by standard mean difference with $95 \%$ confidence interval ( $95 \% \mathrm{Cls}$ ), and dichotomous outcomes measured by odds ratios with $95 \% \mathrm{Cls}$. The statistical

heterogeneity between trial results will be conducted using a $R$ statistic, and $R>50 \%$ will be considered to represent substantial heterogeneity. If $R_{<} 50 \%$, the fixed effects model will be used; while $R>50 \%$, the random effects model will be used. If the quantitative synthesis of data is not possible, qualitative analysis will be applied.

\subsubsection{Subgroup and sensitivity analysis}

We will conduct subgroup analysis to investigate possible sources of heterogeneity across studies such as the stage of diabetic kidney disease, interventions, course of treatment, if sufficient data are available. To validate the robustness of meta-analytic estimates, we will remove each study in turn to observe the impact on the overall results to perform sensitivity analysis.

\subsubsection{Publication bias assessment}

The potential publication bias will be assessed visually using funnel plots and Egger's test, whereas a $p$ value $<0.1$ indicates potential publication bias.

\subsection{Ethics and dissemination}


Ethics approval is not required for this study as patient data will not be involved. We aim to publish the results of this systematic review in a peer-reviewed journal.

\section{Discussion}

It has been reported that the number of patients with CKD is progressively increasing and DKD become the leading cause of ESRD, while other causes of CKD have been relatively stable [12]. The control of glycemic, blood pressure, and LDL-cholesterol level has been demonstrated benefit to DKD, however only in a small proportion of diabetes patients are achieved shown in the real-world evidence study [13]. Chinese medicine has been widely used in DKD and proved to have some benefits in delaying the progress to ESRD. Keluoxin capsule, a proprietary Chinese medicine, could reduce albuminuria, alleviate renal function, and regulate glucose and lipid metabolism in DKD patients. Keluoxin capsule also can relieve glomerular endothelial cell damage, regulate autophagy and protect podocyte [14]. More and more clinical studies suggested that Keluoxin capsule could be a potentially effective treatment for DKD. Thus, it is essential to perform the meta-analysis of published clinical studies to evaluate the efficacy and safety of Keluoxin capsule.

Although the design of systematic review follows Preferred Reported Items for Systematic Review and Meta-analysis guideline, there may still be some limitations. It may lead to regional bias since Keluoxin capsule is mainly used in China. In addition, the included studies also have problems such as small sample size, lacking of follow-up and adverse events reports to make the conclusion uncertain.

\section{Abbreviations}

CBM, Chinese Biomedical Literature Database

DKD, Diabetic kidney disease

DM, Diabetes mellitus

eGFR, Estimated glomerular filtration rate

ESRD, End-stage renal disease

KDOQI, Kidney Disease Outcomes Quality Initiative

RCT, Randomized controlled trials

UACR, Urinary albumin/creatinine ratio

\section{Declarations}

Authors' contributions 
Conceptualization: Jingxin Zhou, Guizhen Miao, Yanbing Gong.

Data curation: Wen Hua, Jie Yang.

Methodology: Qiaoqiao Liu, Yizhen Lu.

Resources: Jian Jin, Jie Yang.

Supervison: Guizhen Miao, Yanbing Gong.

Writing-original draft: Jingxin Zhou, Wenhua Zhang.

Writing-review \& editing: Jingxin Zhou.

All authors read and approved of the final manuscript.

\section{Competing interests}

The authors have declared no financial or commercial conflict of interest.

\section{Ethics approval and Consent to participate}

As the systematic review is based on published studies, ethical considerations are not required. This systematic review and meta-analysis will be published in a peer-reviewed journal.

\section{Consent for publication}

Patient consent for publication is not required.

\section{Funding}

No funding for this protocol.

\section{Acknowledgements}

This work was supported by the Youth Qihuang Scholar Support Project of the State Administration of Traditional Chinese Medicine (National Traditional Chinese Medicine People's Education Development [2020] No. 7).

\section{References}

1. International Diabetes Federation (IDE). Diabetes Atlas, 9th ed.; International Diabetes Federation: Brussels, Belgium, 2019.

2. Kato $M$, Natarajan R. Epigenetics and epigenomics in diabetic kidney disease and metabolic memory. Nat Rev Nephrol. 2019;15(6):327-345. 
3. Ma CE, Yu P, Wei W, Chen XQ. Efficacy of combined angiotensin II receptor blocker with tripterygium glycosides on diabetic nephropathy: A protocol for meta-analysis. Medicine (Baltimore). 2021;100(22):e25991.

4. Pugliese G, Penno G, Natali A, et al. Diabetic kidney disease: new clinical and therapeutic issues. Joint position statement of the Italian Diabetes Society and the Italian Society of Nephrology on "The natural history of diabetic kidney disease and treatment of hyperglycemia in patients with type 2 diabetes and impaired renal function". J Nephrol. 2020;33(1):9-35.

5. Lytvyn Y, Bjornstad P, van Raalte DH, Heerspink HL, Cherney DZI. The New Biology of Diabetic Kidney Disease-Mechanisms and Therapeutic Implications. Endocr Rev. 2020;41(2):202-231.

6. Fitchett D. A safety update on sodium glucose co-transporter 2 inhibitors. Diabetes, Obesity and Metabolism. 2019;21(S2):34-42.

7. Zhang L, Yang L, Shergis J, Zhang L, Zhang AL, Guo X, Qin X, Johnson D, Liu X, Lu C, Xue CC, Mao W. Chinese herbal medicine for diabetic kidney disease: a systematic review and meta-analysis of randomised placebo-controlled trials. BMJ Open. 2019;9(4):e025653.

8. Wu R, Wei F, Qu L, Bai L, Li J, Li F, Yan W, Wang Q, Wei J. Effects of Keluoxin capsule combined with losartan potassium on diabetic kidney disease: study protocol for a randomized double-blind placebo-controlled multicenter clinical trial. Trials. 2020;21(1):951..

9. Shamseer L, Moher D, Clarke M, Ghersi D, Liberati A, Petticrew M, Shekelle P, Stewart LA; PRISMA-P Group. Preferred reporting items for systematic review and meta-analysis protocols (PRISMA-P) 2015: elaboration and explanation. BMJ. 2015;350(1):g7647.

10. National Kidney Foundation. KDOQI Clinical Practice Guideline for Diabetes and CKD: 2012 Update.National Kidney Foundation. Am J Kidney Dis. 2012;60(5):850-86.

11. Higgins J, Green S. Cochrane handbook for systematic reviews of interventions version 5.1.0; 2011. Retrieved October 8, 2020.

12. Thomas, B. The Global Burden of Diabetic Kidney Disease: Time Trends and Gender Gaps. Curr Diab Rep. 2019;19(4): 18.

13. Gembillo G, Ingrasciotta Y, Crisafulli S, Luxi N, Siligato R, Santoro D, Trifirò G. Kidney Disease in Diabetic Patients: From Pathophysiology to Pharmacological Aspects with a Focus on Therapeutic Inertia. Int J Mol Sci. 2021;22(9):4824.

14. Yang X, Han X, Wen Q, Qiu X, Deng H, Chen Q. Protective Effect of Keluoxin against Diabetic Nephropathy in Type 2 Diabetic Mellitus Models. Evid Based Complement Alternat Med. 2021:8455709. 